\title{
Classic yin and yang tonic formula for osteopenia: study protocol for a randomized controlled trial
}

Feng Yang ${ }^{1,2}$, De-Zhi Tang ${ }^{1,3^{*}}$, Xue-Jun Cui ${ }^{1,3}$, Jonathan D Holz ${ }^{4,5}$, Qin Bian ${ }^{1,3}$, Qi Shi ${ }^{1,3}$ and Yong-Jun Wang ${ }^{1,3^{*}}$

\begin{abstract}
Background: Osteoporosis is a growing worldwide problem, with the greatest burden resulting from fractures. Nevertheless, the majority of fractures in adults occur in those with "osteopenia" (bone mineral density (BMD) only moderately lower than young normal individuals). Since long-term drug therapy is an expensive option with uncertain consequences and side effects, natural herbal therapy offers an attractive alternative. The purpose of this study is to evaluate the effect on BMD and safety of the Classic Yin and Yang Tonic Formula for treatment of osteopenia and to investigate the mechanism by which this efficacy is achieved.

Methods/design: We propose a multicenter double-blind randomized placebo-controlled trial to evaluate the efficacy and safety of the Classic Yin and Yang Tonic Formula for the treatment of osteopenia. Participants aged 55 to 75 with low bone mineral density (T-score between -1 and -2.5) and kidney deficiency in TCM will be included and randomly allocated into two groups: treatment group and control group. Participants in the treatment group will be treated with Classic Yin and Yang Tonic Granule, while the controlled group will receive placebo. Primary outcome measure will be BMD of the lumbar spine and proximal femur using dual-energy $\mathrm{X}$-ray absorptiometry. Secondary outcomes will include pain intensity measured with visual analogue scales, quality of life, serum markers of bone metabolism, indices of Neuro-endocrino-immune network and safety.

Discussion: If the Classic Yin and Yang Tonic Formula can increase bone mass without adverse effects, it may be a novel strategy for the treatment of osteoporosis. Furthermore, the mechanism of the Chinese medical formula for osteoporosis will be partially elucidated.

Trial registration: This study is registered at ClinicalTrials.gov, NCT01271647.
\end{abstract}

\section{Background}

Osteoporosis is a growing problem worldwide, with the greatest burden resulting from fractures. Nevertheless, the majority of fractures in adults occur in those with "osteopenia" (bone mineral density (BMD) only moderately lower than young normal individuals). Thus, there has been an interest in developing approaches to prevent bone loss. The agents currently approved for treatment and/or prevention of osteopenia include bisphosphonates, hormone replacement therapy with estrogen or combination estrogen/progesterone preparations, calcitonin, raloxifene, and parathyroid hormone as well. But

\footnotetext{
* Correspondence: dztang702@hotmail.com; yjwang88@hotmail.com 'Longhua Hospital, Shanghai University of Traditional Chinese Medicine, Shanghai, 200032, PR China Full list of author information is available at the end of the article
}

short- and long-term consequences (adverse effects) of these therapies continue to be discovered. The adverse outcomes include cancer, and cardiac, dermatologic, gastrointestinal, gynecologic, immunologic, metabolic, musculoskeletal, neurological, psychiatric, and respiratory events.

Since long-term drug therapy is an expensive option with uncertain consequences and side effects, natural herbal therapy offers a possible alternative. The Classic Yin and Yang Tonic Formula (CYYTF) have been used to treat osteoporosis or osteopenia in traditional Chinese medicine (TCM) for a long time. Several putative mechanisms for this have been proposed. These include stimulation of osteoblast proliferation and differentiation, osteogenesis, and inhibition of bone resorption, as a estrogen-like function. For this reason, Chinese guidelines for
C Biomed Central 
the treatment of osteopenia include natural herbal therapy. However, there is currently no randomized placebocontrolled trial to verify its efficacy in treating bone mass loss. The present study is to examine effects of a Chinese herbal intervention using the Classic Yin and Yang Tonic Formula (CYYTF) to improve bone mineral density of patients with osteopenia in a randomized, double-blind, placebo-controlled trial. Results of this study will provide evidence regarding the value of the Classic Yin and Yang Tonic Formula (CYYTF) as an intervention to increase BMD of osteopenic individuals. Furthermore, the mechanisms of action can be partially identified by this study.

\section{Methods and Design Study Design}

This clinical trial is a multi-center, randomized, doubleblind, placebo-controlled design. Subjects will be enrolled at four hospitals: 1) Longhua hospital affiliated with Shanghai university of TCM; 2) Huadong hospital affiliated with Fudan university; 3) The central hospital of Yangpu district, Shanghai; 4) The Red Cross hopital of Xi'an. This study is conducted in 4 sites in China.

\section{Ethics Issue}

This study has been approved by the Ethics Board of Shanghai University of TCM (No: 2011LCSY002). Each participating centre obtained a local Institutional Review Board Approval. All study participants will sign the written informed consent prior to participation.

\section{Patients Population and Recruitment Procedure}

The study population consists of individuals aged 55 to 75 with BMD below $80 \%$ of the young adult mean (YAM) (T score -1.0 to -2.5 at the lumbar spine or hip). Data for the YAM and T score values were obtained from reference data of healthy women 20 to 29 years of age [1]. The individuals whose syndrome differentiation are kidney deficiency will be included. In traditional Chinese medicine (TCM), kidney deficiency syndrome is a general term for deficiency conditions of kidney and Neuro-endocrinoimmune network, The symptoms mainly include: low back pain, soreness and weakness of the lumbar regions and knees, dizziness, fatigue, spontaneous sweating, a hot or cold sensation in the palms, soles and chest, dysphoria, insomnia, The pulse is weak, the tongue is red and covered without fur or bulky, moist, and covered with white fur.

Subjects will be excluded if they have disorders such as primary hyperparathyroidism; Cushing's syndrome; premature menopause due to hypothalamic, pituitary, or gonadal insufficiency; poorly controlled diabetes mellitus (HbA1c $>8.0 \%$ ); or other causes of secondary osteoporosis. Subjects will also be excluded if they have taken bisphosphonates at any time. The trial will exclude individuals who have taken glucocorticoids, calcitonin, vitamin $\mathrm{K}$, active vitamin $\mathrm{D}$ compounds, or hormone replacement therapy within the previous 2 months. In addition, serum calcium (Ca) levels above $10.6 \mathrm{mg} / \mathrm{dL}(2.7 \mathrm{mmol} / \mathrm{L})$ or below $8.0 \mathrm{mg} / \mathrm{dl}(2.0 \mathrm{mmol} / \mathrm{L})$, serum creatinine levels above1.5 mg/dL $(133 \mu \mathrm{mol} / \mathrm{L})$, or clinically significant hepatic disorders, are also exclusion. Furthermore, subjects that have Malignancies, or physical or mental disabilities will be excluded, as well as lactating or pregnant patients.

This study is to be conducted in accordance with protection of patients, as outlined in the Declaration of Helsinki, and approved by the appropriate Institutional Review Boards. Each participant will sign the written informed consent before undergoing any examination or study procedure, in compliance with Good Clinical Practice. We will utilize EpicCare databases to identify all persons aged 55-75 who had a DXA scan during the prior two years at any of the four hospitals, and with BMD of the hip (femoral neck or trochanter) and/or spine falling within the T-score range of -1.0 and -2.5 . The patients whose syndrome differentiation of TCM is kidney deficiency are included. Patients who initially meet these eligibility criteria are then to complete the additional baseline testing and will be randomized into either the treatment or the control group.

\section{Interventions}

Eligible patients will be randomized to one of the two arms: placebo and Chinese medical herb (18 g twice daily). All drugs will be administered orally for 6 months. In this study, the treating physicians, subjects, investigators and statisticians will be blinded to treatment assignment. In this respect, the trial is double-blind. Randomization of subjects will occur centrally using a random number generator and will be stratified by syndrome differentiation of TCM (Syndrome, as related to illnesses in Western medicine, is composed of a set of signs and/or symptoms classified by Traditional Chinese Medicine practitioners [2]. TCM Syndrome differentiation is based on symptoms and helps to identify a subset of disease). Patients visit the doctor at 6 and 12 months follow-up.

\section{Randomization and Allocation}

Treatment allocation occurs when the study participant meets the inclusion criteria and signs the informed consent form. The teletherapist will then register the participant into the database, which in turn asks if they are ready to be randomized. After the teletherapist enters yes, the site-specific randomization program behind the form displays the participant's group assignment number (placebo versus Chinese medical herb). Figure 1 shows the process of patient enrollment and allocation. Study participants will be randomized in blocks of random sizes 


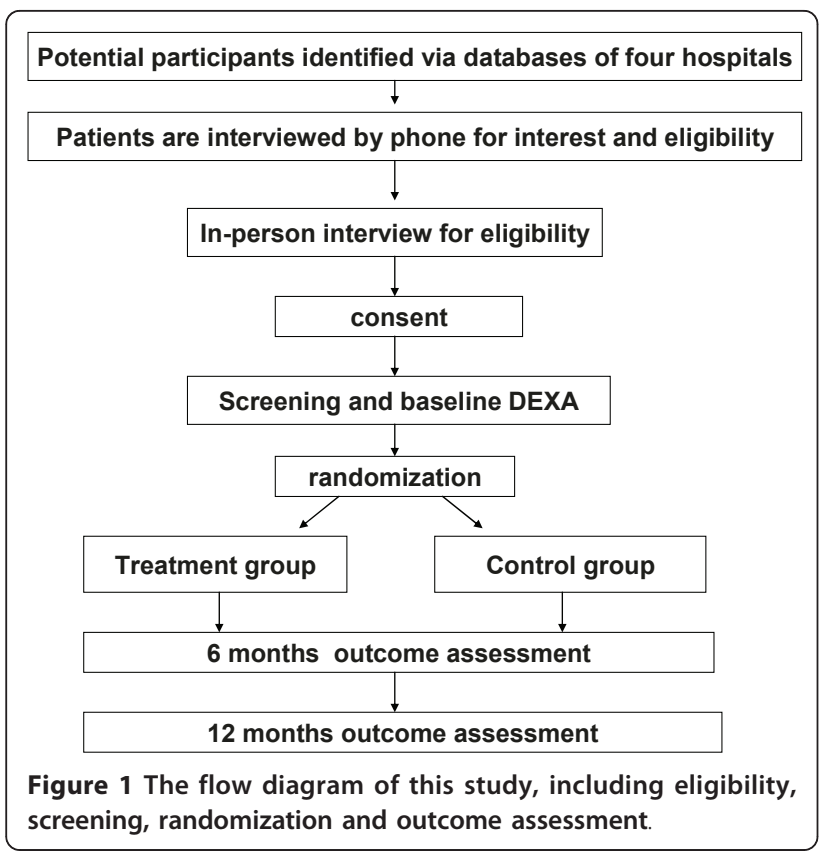

of 2 and 4 at each site. Site-specific randomization lists will be computer-generated (i.e., generated by an individualized basic visual code program) and concealed from the researchers by a senior data manager who is not involved in the study. This information will remain confidential and is not shared with the study sites, in concordance with the CONSORT guidelines. This trial uses a prospective, randomized, outcome-blinded design, in which all outcome assessments are made by a research assistant blinded to treatment allocation and uninvolved in patient consent and management.

\section{Outcome Measurements}

The primary outcome of the study is bone mineral density at 6 and 12 months after treatment with the study medication. Secondary outcomes include pain intensity, quality of life, relative changes in bone turnover markers, neuro-endocrino-immune indices, and safety.

\section{Assessment of bone mineral density (BMD)}

Lumbar spine BMD (L1-4) and femoral BMD will be measured at three measurement points (baseline and 6 and 12 months after the treatment), using the dual energy X-ray absorptiometry densitometer obtained from Hologic, Inc. USA. The accuracy of the measurements recorded by the dual energy X-ray absorptiometry (DXA) instruments is to be evaluated by the use of serial measurements of a local spine "phantom". The variability of DXA measurements across the different participant centres will also be assessed via utilization of the same spine "phantom" technique. Using this technique, the long-term coefficient of variation of each instrument in the study is estimated as less than $1 \%$. These "phantom" measurements will be used to adjust for any "drift" in measurements of bone densitometry during the study.

\section{Assessment of bone turnover}

Serum samples will be collected at baseline and 6 months after the treatment for measurement of bone turnover markers, including type I collagen N-telopeptide (NTX), serum bone-specific alkaline phosphatase (BALP), serum osteocalcin (OC), serum Procollagen type I N-terminal propeptide (PINP), carboxy-terminal telopeptide of type-I collagen (CTX), and Serum procollagen I carboxy-terminal propeptide (PICP).

\section{Health-related quality of life}

The ECOS-16 questionnaire is developed with the aim of measuring health related quality of life (HRQoL) in postmenopausal women with osteoporosis. It is based on the combination of two disease-specific HRQoL questionnaires for women with osteoporosis: the Osteoporosis Quality of Life Questionnaire (OQLQ) [3] and the Quality of Life Questionnaire of the European Foundation for Osteoporosis (QUALEFFO) [4]. The 16 items in the new questionnaire are divided qualitatively into four dimensions. The nature of the four dimensions also suggests that they can be further combined to produce two summary scores that would include Physical Function and Pain in one Physical score and another that would include Fear of Illness and Psychosocial Function in a Mental score. These two summary scores could, in turn, be combined to provide an overall score for the questionnaire. However, although the 16 items can be classified qualitatively into four dimensions, this is a unidimensional questionnaire, according to quantitative analysis [5]. The score of each item ranges from 1 to 5 . The ECOS-16 questionnaire generates a single summary score obtained from the arithmetic mean of the answered items, so the total score ranges from 1 (best HRQoL) to 5 (worst HRQoL). This questionnaire will be completed at all the measurement points (baseline and 6 and 12 months after the treatment).

\section{Assessment of pain}

The Visual Analogue Scale (VAS) measures amount of pain, which is a pain score ranging from 0 (no pain) to 100 (worst pain ever) [6]. Operationally, the VAS score is usually a horizontal line, $100 \mathrm{~mm}$ in length, anchored by word descriptors at each end. The patient marks on the line the point that they feel represents their perception of their current pain. The VAS score is then determined by measuring in millimetres from the left hand end of the line to the point that the patient marks. The VAS score will be measured at all the measurement points (baseline and 6 and 12 months after the treatment). 


\section{Assessment of adverse events}

All subjects are to be questioned about adverse events (AEs) during treatment at each visit point, and all adverse events reported will be analyzed regardless of the investigators' assessments of causality. The Medical Dictionary for Regulatory Activities (MedDRA, Version 8.1J) will be used to categorize reported adverse events.

\section{Sample Size Considerations}

We calculate the required sample size for this trial based on the following calculation: $\mathrm{n}=2 \sigma^{2} \times f(\alpha, \beta) /\left(\mu_{1}-\mu_{2}\right)^{2}$ [7]. First, we estimate that an absolute improvement of $7.8 \%$ (from $\mu_{1}$ to $\mu_{2}, \mu_{1}=0.803, \mu_{2}=0.866$ ) in BMD was likely the smallest clinically-relevant difference [8]. Second, we assume that standard deviation of BMD may be $0.125(\sigma=$ 0.125 ) at baseline[9]. Based on these assumptions, we will require 84 patients in each group to have at least a $90 \%$ power $\beta=0.1$ and to rule out a two-sided type I error of $5 \% \alpha=0.05$. This number of patients actually provides less than $80 \%$ power, assuming an withdrawal rate of $20 \%$. Therefore, we will recruit approximately a total of 204 patients, 102 patients in each group.

\section{Statistical Analysis}

The data will be collected and analysed according to the intention-to-treat principle. Standard statistical techniques will be used to describe characteristics of patients in both groups. We will compare baseline characteristics in both groups and if incomparability appears, we will perform the secondary analysis, adjusting for differences. The primary outcome, BMD, will be compared between both groups using analysis of variance for repeated measures. If adjustment for possible baseline incomparability is needed, analysis of covariance will be done.

\section{Discussion}

Osteoporosis is a major cause of morbidity and mortality in older persons. For individuals identified to be at risk for developing osteoporosis, intervention measurements are aimed at preventing the condition from getting worse, whereas in those who have already presented with low BMD or fractures, treatment is aimed at preventing further bone loss to reduce the risk of initial or subsequent fracture. Currently, there is no good evidence that these BMD differences may then translate in fracture reduction changes. However, Low bone mineral density (BMD) is a known risk factor of fracture and a strong predictor of new vertebral fracture $[10,11]$. A previous publication reported that after the adjustment for age and BMI, the odds of having a vertebral fracture in Southern Chinese women was 2.3(1.6-3.3) for each $1 \mathrm{SD}$ reduction in spine BMD and 2.1(1.37-3.20) for femoral neck BMD [12]. A latest publication revealed that women with prevalent vertebral fractures had significantly lower BMD than those without prevalent vertebral fractures[13]. Therefore, we use BMD as the primary outcome.

Estrogens have important anticatabolic and anabolic effects on bone, and estrogen deficiency plays a central role in the development of osteoporosis [14]. Hormone replacement therapy (HRT) effectively prevents bone loss in postmenopausal women $[15,16]$ and reduces the incidence of fractures[17,18]. However, long-term acceptance or compliance of HRT is generally poor in postmenopausal due to the potential complications, such as uterus hyperplasia and breast cancer $[19,20]$. A recent review suggested that many non-leguminous plants are rich in phytoestrogen, which is regarded as a prospective candidate for treatment of postmenopausal osteoporosis [21]. Among non-leguminous plants, Epimedium brevicornum maxim is a centuries-old medicinal herb. It is reputed by Traditional Chinese Medicine to have "bone strengthening" function and used for treatment of musculoskeletal disorders [22]. Epimedium-derived phytoestrogen flavonoids (EPFs) have been shown to prevent bone loss in late postmenopausal women, as indicated by the significant maintenance of BMD in the EPF treatment group compared to the placebo control group [23]. In this study, the Classic Yin and Yang Tonic Formula contains diverse phytoestrogen, such as isoflavone, lignans and coumarin. If this study demonstrates the effectiveness and safety of the Classic Yin and Yang Tonic Formula significant strides would be made towards a clinically useful therapy for reducing bone mass loss.

List of abbreviations

BMD: (Bone mineral density); DXA: (Dual energy X-ray absorptiometry); CYYTF: (Classic Yin and Yang Tonic Formula); VAS: (Visual Analogue Scale); TCM: (Traditional Chinese Medicine); YAM: (Young adult mean); EPFs: (Epimedium-derived phytoestrogen flavonoids); NTX: (Type I collagen Ntelopeptide); BALP: (Bone-specific alkaline phosphatase); OC: (Osteocalcin); PINP: (Procollagen type I N-terminal propeptide); CTX: (Carboxy-terminal telopeptide of type-I collagen); PICP: (Procollagen I carboxyterminal propeptide); OQLQ: (Osteoporosis Quality of Life Questionnaire); HRQoL: (Health related quality of life); QUALEFFO: (Quality of Life Questionnaire of the European Foundation for Osteoporosis).

\section{Acknowledgements}

This work was supported in part by the National Basic Research Program of China (973 Program, 2010 CB530400 to YW) and the Key Program of Natural Science Foundation of China (30930111 to YW).

\section{Author details}

1'Longhua Hospital, Shanghai University of Traditional Chinese Medicine, Shanghai, 200032, PR China. ${ }^{2}$ Shaanxi University of Traditional Chinese Medicine, Xianyang, 712000, PR China. ${ }^{3}$ Spine Research Institute, Shanghai University of Traditional Chinese Medicine, Shanghai, 200032, PR China. ${ }^{4}$ Department of Orthopaedics, Center for Musculoskeletal Research, University of Rochester, Rochester, New York 14642, USA. ${ }^{5}$ Department of Environmental Medicine, University of Rochester, Rochester, New York 14642, USA.

\section{Authors' contributions}

FY, DZT, XJC, QB, YJW, QS contributed to the design of the study. All authors contributed to the creation of the Manual of Procedures, implementation of the study protocol and acquisition of data. FY, DZT and 
XJC drafted the manuscript, and JDH modified the manuscript. All authors provided critical revision and approved the final manuscript.

\section{Competing interests}

The authors declare that they have no competing interests.

Received: 27 February 2011 Accepted: 2 August 2011

Published: 2 August 2011

\section{References}

1. Woo J, Ji M, Lau E: Population bone mineral density measurements for Chinese women and men in Hong Kong. Osteoporos Int 2001, 12:289-295.

2. Guang JY: The mode of thinking in Chinese clinical medicine: characteristics, steps and forms. Clin Acupunct Orient Med 2001, 2:23-28.

3. Lips P, Cooper C, Agnusdei D, et al: Quality of life in patients with vertebral fractures: validation of the Quality of Life Questionnaire of the European Foundation for Osteoporosis (QUALEFFO). Working Party for Quality of Life of the European Foundation for Osteoporosis. Osteoporos Int 1999, 10(2):150-160.

4. Badia X, Díez-Pérez A, Alvarez-Sanz C, Díaz-López B, Díaz-Curiel M, Guillén F, Gonzalez-Macias J, Spanish GRECO Study Group: Measuring quality of life in women with vertebral fractures due to osteoporosis: A comparison of the OQLQ and QUALEFFO. Qual Life Res 2001, 10:307-317.

5. Badia X, Prieto L, Roset M, Herdman M: Development of a short osteoporosis quality of life questionnaire by equating items from two existing instruments. J Clin Epidemiol 2002, 55:32-40.

6. Gould D, et al: Visual Analogue Scale (VAS). Journal of Clinical Nursing 2001, 10:697-706.

7. Pocock SJ: Clinical trials: A practical approach. Chichester: John Wiley Sons Ltd. England; 1 1983, 123-141.

8. Liu GY, Qiu GY, Wu ZH: Racial differences in peak bone mass assessed through NORLAND DXA. Chin J Osteoporosis 2007, 13(2):101-103.

9. Wei SQ, Yang DZ, Wu T, et al: Evaluation of the efficacy and safety of domestic oral alendronate in the treatment of postmenopausal osteoporosis. Chin J Endocrinol Metab 1998, 14:348-350.

10. Kanis JA, Johnell O, Oden A, Borgstrom F, Zethraeus N, De Laet C, Jonsson B: The risk and burden of vertebral fractures in Sweden. Osteoporos Int 2004, 15(1):20-26.

11. Cauley JA, Hochberg MC, Lui LY, Palermo L, Ensrud KE, Hillier TA, Nevitt MC, Cummings SR: long-term risk for new fracture. JAMA 2007, 298(23):2761-2767.

12. Kung AW, Luk KD, Chu LW, Tang GW: Quantitative ultrasound and symptomatic vertebral fracture risk in Chinese women. Osteoporos Int 1999, 10:456-461.

13. Cauley JA, Palermo L, Vogt M, Ensrud KE, Ewing S, Hochberg M, Nevitt MC, Black DM: Prevalent vertebral fractures in black women and white women. J Bone Miner Res 2008, 23:1458-1467.

14. Raisz LG: Pathogenesis of osteoporosis: concepts, conflicts, and prospects. J Clin Invest 2005, 115:3318-3325

15. Lindsay R, Hart DM, Aitken JM, MacDonald ED, Anderson JB, Clarke AC: Long-term prevention of postmenopausal osteoporosis by oestrogen. Lancet 1976, 1:1038-1041

16. Lindsay R, Hart DM, Clark DM: The minimum effective dose of estrogen for prevention of postmenopausal bone loss. Obstet Gynecol 1984, 63:759-763.

17. Gordon GS, Picchi J, Roof BS: Antifracture efficacy of long-term oestrogens for osteoporosis. Trans Assoc Am Physicians 1973, 86:326-331.

18. Hutchinson TA, Polansky SM, Feinstein AP: Postmenopausal estrogens protect against fractures of hip and distal radius. Lancet 1979, 2:706-709.

19. Michaelsson K, Baron JA, Farahmand BY, Johnell O, Magnusson C, Persson PG, Persson I, Ljunghall S: Hormone replacement therapy and risk of hip fracture: Population based case-control study. The Swedish Hip Fracture Study Group. BMJ 1998, 316:1858-1863.

20. Blank RD, Bockman RS: A review of clinical trials of therapies for osteoporosis using fracture as an end point. J Clin Densitom 1999, 2:435-452.

21. Mackova Z, Koblovska R, Lapcik O: Distribution of isoflavonoids in nonleguminous taxa - an update. Phytochemistry 2006, 67:849-855

22. Qin L, Zhang G, Shi Y, Lee KM, Leung PC: Prevention and treatment of osteoporosis with traditional herbal medicine. In Current Topics of
Osteoporosis. Edited by: Deng HW, Liu YZ, Guo CY, Chen D. World Scientific Publisher, Singapore; 2005:513-531.

23. Zhang G, Qin L, Shi Y: Epimedium-Derived Phytoestrogen Flavonoids Exert Beneficial Effect on Preventing Bone Loss in Late Postmenopausal Women: A 24-Month Randomized, Double-Blind and Placebo-Controlled Trial. J Bone Miner Res 2007, 22(7):1072-1079.

doi:10.1186/1745-6215-12-187

Cite this article as: Yang et al:: Classic yin and yang tonic formula for osteopenia: study protocol for a randomized controlled trial. Trials 2011 $12: 187$.

\section{Submit your next manuscript to BioMed Central and take full advantage of:}

- Convenient online submission

- Thorough peer review

- No space constraints or color figure charges

- Immediate publication on acceptance

- Inclusion in PubMed, CAS, Scopus and Google Scholar

- Research which is freely available for redistribution
C Biomed Central 